Landero Amaya, J. A., Carrasco Ruiz, P. M., \& González, M. (2021). Evaluación de dos métodos de sincronización de estro e inseminación artificial transcervical en capra aegagrus hircus, Estelí 2020. Teknos Revista Cientifica, 21(2), 17-23.

\title{
Evaluación de dos métodos de sincronización de estro e inseminación artificial transcervical en capra aegagrus hircus, Estelí 2020
}

\section{Evaluation of two methods of synchronization of estrus and transcervicall artificial insemination in capra aegagrus hircus, Estelí 2020}

\section{Jaime Antonio Landero Amaya1, Paola Marcela Carrasco Ruiz², González M}

${ }^{1}$ Docente Investigador de la facultad de ciencias agropecuaria Universidad Católica del Trópico Seco - Estelí Nicaragua jlamaya63@yahoo.com; alicia@ucatse.edu.ni

2 Médico Veterinario egresado. UCATSE. Estelí Nicaragua. paovet20@gmail.com

Recibido: 15/ago/2021 Revisado: 30/sep/2021

Aceptado: 30/oct/2021 Publicado: 30/dic/2021

Resumen La presente investigación se realizó en la Universidad Católica del Trópico Seco, ubicada en Estelí, Nicaragua. El objetivo fue evaluar dos métodos de sincronización de estro con dispositivos horm onales CIDR nuevos y reutilizados, para inseminación artificial transcervical en cabras (Capra aegagrus hircus). Se utilizó un diseño cuasi experimental comparativo. Dentro de los tratamientos utilizados están: T1 sincronización de estro con dispositivos hormonales CIDR nuevos para la inseminación artificial con semen fresco, T2 con CIDR reutilizados para la insemina ción artificial. En la variable Efectividad de los métodos de sincronización de estro, se aplicó una prueba T de Student, donde los resultados obtenidos no tuvieron diferencias estadísticas. En la variable porcentaje de preñez, no se presentó diferencia estadística. En la variable beneficio/costo el T1 resalta la confiabilidad en el componente rentabilidad obteniendo un 1.04 con el análisis RCB. Los resultados del análisis de la variable: Propuesta de plan de manejo reproductivo en UCATSE, se concluye que se deben reestablecer ámbitos de manejo nutricional y de instalaciones para la implementación en la reproducción caprina utilizando los métodos de sincronización del estro e inseminación artificial, para la obtención del incremento en la producción caprina.

Palabras claves sincronización del estro; inseminación artificial; Capra aegagrus hircus; preñez; reproducción.

Abstrac This research was conducted at the Catholic University of the Dry Tropics, located in Estelí, Nicaragua. The objective was to evaluate two methods of estrus synchronization with new and reused CIDR hormonal devices for transcervical artificial insemination in goats (Capra aegagrus hircus). A comparative quasi-experimental design was used. Among the treatments used are: T1 estrus synchronization with new CIDR hormonal devices for artificial insemination with fresh semen, T2 with reused CIDR for artificial insemination. In the variable Effectiveness of estrus synchronization methods, a Student's T test was applied, where the results obtained had no statistical differences. In the variable percentage of pregnancy, there was no statistical difference. In the benefit/cost variable, T1 highlights the reliability in the profitability component, obtaining a 1.04 with the RCB analysis. The results of the analysis of the variab le: Proposal of reproductive management plan in UCATSE, it is concluded that areas of nutriti onal management and facilities for the implementation in goat reproduction using the methods of synchronization of estrus and artificial insemination should be reestablished, to obtain the increase in goat production.

Keywords Estrus synchronization; Artificial insemination; Capra aegagrus hircus; pregnancy; reproduction.

Teknos Revista Científica. | Volumen 21 No.2 - diciembre 2021 | ISSN 1900-7388 (papel) | ISSN 2539-2190 (digital)

DOI: https://doi.org/10.25044/issn.2539-2190 


\section{Introducción}

Las explotaciones caprinas de Nicaragua se han llevado en forma tal que no han permitido determinar la función zootécnica ordenada y el trabajo de mejora genética más adecuado para nuestro medio y para las razas que se encuentran en el país, que son pocas. Nicaragua reúne las características necesarias para el desarrollo caprino en gran escala, ya que cuenta con grandes extensiones de zonas secas (suelos pedregosos, poco profundos y de topografía variable), en donde las explotaciones de ganado mayor son cada día menos rentables, sin embargo, existe el problema de contar con una explotación caprina demasiado baja en producción (Sáenz García, 2007).

El factor principal en la problemática del desarrollo caprino de Nicaragua es la cultura en el manejo productivo de cabras, por ende, las demás acciones de manejo como la reproductiva, que es una de las más importantes en un área caprina, se ven afectadas y no hay producción efectiva en la caprinocultura del país. Resulta de gran importancia poder establecer métodos seguros para la sincronización de estros en las cabras, teniendo en cuenta la importancia del bienestar animal, el beneficio ecológico y el beneficio económico para efectuar la inseminación artificial de la mejor manera, obteniendo resultados positivos en la reproducción caprina del país.

La presente investigación surge de la necesidad del estudio de dos métodos de sincronización de estro con dispositivos hormonales y la inseminación artificial transcervical en las cabras, de la Universidad Católica del Trópico Seco, en donde se verán beneficiados el productor por los índices de reproducción animal, teniendo en cuenta que, durante la ejecución, se evitarán infecciones vaginales por el tipo de dispositivo a utilizar, se disminuirán los costos con la reutilización del dispositivo para sincronizar el celo en las cabras y de igual manera el método a utilizar en la inseminación artificial, será el menos traumático e invasivo para cada animal. La investigación busca generar principalmente la producción de la especie caprina y por ende, la obtención de carne, leche y los derivados lácteos como subproductos.

En los sistemas de producción caprina, la reproducción es uno de los factores determinantes en la organización del caprinocultor, del manejo de esta, dependerá la programación de prácticamente todas las demás acciones de manejo, por lo tanto, las variables que puedan incidir deben ser analizadas exhaustivamente, buscando ajustar cada detalle para lograr los objetivos planteados. (De la Rosa, 2011).

La sincronización del ciclo estral permite la aparición de celo en determinado momento, para realizar la monta natural o inseminación artificial y agrupar nacimientos (Molina, 2005).

Los compuestos más comunes para la sincronización en ovino-caprinos es el uso de progestágenos como el acetato de fluorogestona (FGA) y el acetato de medroxiprogesterona (MAP). Todos ellos son aplicados intravaginalmente, ya sea en forma de un dispositivo de liberación (CIDR) o en forma de esponjas de poliuretano alrededor de 12-14 días (Contreras, 2008).

La inseminación artificial cervical es a la fecha la más utilizada, la deposición del semen se realiza dentro de los pliegues cervicales, los cuales son visibles con la ayuda de un espéculo y fuente de luz para depositar el semen intrauterinamente, la técnica implica la sujeción del cérvix por la vagina para permitir la introducción del instrumento inseminatorio en el canal cervical.

Se ha demostrado que la inseminación artificial intrauterina por laparotomía exploratoria es invasiva, ya que la técnica incluye una ligera sedación del animal para permitir la laparotomía la cual incluye la exposición de ambos cuernos para la deposición del semen fresco o congelado/descongelado con un trocar o catéter. (Asociación Cooperativa La Galea, 2012).

El objetivo de la presente investigación fue la evaluación de dos métodos de sincronización de estro e inseminación artificial en cabras (Capra aegagrus hircus), con el propósito de dar conocer a los ganaderos del sector caprino y a los estudiantes de la

Teknos Revista Científica. | Volumen 21 No.2 - diciembre 2021 | ISSN 1900-7388 (papel) | ISSN 2539-2190 (digital)

DOI: https://doi.org/10.25044/issn.2539-2190 
facultad de ciencias agropecuarias, la importancia de utilizar protocolos de sincronización de celos e inseminación artificial con métodos seguros, garantizando el bienestar animal y el beneficio que trae para un manejo reproductivo más eficiente en los hatos, y un mayor desarrollo de los caprinocultores de Nicaragua y Centroamérica.

El trabajo realizado por (Mogedas Moreno, 2016) "Sincronización de la ovulación y el ciclo inducido por el efecto "macho" mediante la administración de progesterona por vía intravaginal en cabras en período de anestro estacional", obtuvo los siguientes resultados: fertilidad en 228 cabras inseminadas, las cuales no mostraron diferencias significativas con ambos métodos utilizados, que fueron $57,25 \%$ intravaginal vs $48,8 \%$ intramuscular.

En el estudio de (Enríquez Palos, 2017) "Porcentaje de fertilidad de ovejas con estro sincronizado con CIDR reciclado y retirado en tres periodos de tiempo a través de inseminación artificial por laparoscopía", se emplearon 30 ovejas que se inseminaron artificialmente por laparoscopía, realizado a partir de las 52 horas de retirados los CIDR. Se concluyó, que el uso de CIDR reciclados son tan efectivos como el uso CIDR nuevos o de esponjas intravaginales de acetato de fluorogestena en relación al porcentaje de gestación y fertilidad en ovejas inseminadas artificialmente por laparoscopía, además de influir directamente en la disminución de los costos de la sincronización del estro con el uso de CIDR reciclados.

\section{Metodología}

La presente investigación se realizó en el módulo caprino de la Finca Santa Adelaida situada en la Universidad Católica del Trópico Seco (UCATSE), ubicada en el kilómetro $1661 / 2$ de la carretera Panamericana norte, a los $13^{\circ} 14^{\prime} 52^{\prime \prime}$ de latitud norte y $86^{\circ} 22^{\prime} 37^{\prime \prime}$ de longitud oeste, con una precipitación promedio de $923 \mathrm{~mm}$ anuales, presentando una humedad relativa del $57 \%$ al $78 \%$ y una temperatura media anual de $21.4{ }^{\circ} \mathrm{C}$, catalogada bajo condiciones de trópico seco.
La muestra estuvo conformada por 10 cabras, seleccionadas en dos grupos de 5 y se distribuyeron en dos tipos de tratamientos de sincronización de estro para la inseminación artificial. Para este estudio se implementó la observación, la cual estuvo detallada en una hoja de campo que midió variables como, los cambios en la etiología y en el tamaño de la vulva de las cabras previas a entrar al estro, el estado sanitario del animal y del medio donde se encuentran, el estado reproductivo de cada una y el estado nutricional basado en el tipo de alimentación que se les proporcione. Además de incluir ambos tratamientos abreviados como T1 Y T2.

T1: Se refiere al método de sincronización del estro con dispositivos CIDR nuevos y la utilización de tres tipos de hormonas PG, eCG y $\mathrm{GnRH}$ para la inseminación artificial transcervical en Capra aegagrus hircus.

T2: Se refiere al método de sincronización del estro con dispositivos CIDR reutilizados por segunda vez y la utilización de tres tipos de hormonas PG, eCG y $\mathrm{GnRH}$ para la inseminación artificial transcervical en Capra aegagrus hircus.

Se realizó un diseño cuasi experimental comparativo, en el cual se trabajó con dos grupos experimentales con 5 unidades de análisis para cada uno, bajo condiciones estabuladas, divididas en dos tipos de tratamiento que permitieron realizar la evaluación del estro y la determinación del porcentaje de preñez en las cabras inseminadas.

Los datos numéricos se introdujeron en una base de datos en Microsoft Excel, que luego se incorporaron en InfoStat v10, se procedió a realizar una prueba de normalidad de Kolmogorov y posteriormente se ejecutó un análisis de $\mathrm{T}$ de Student al $95 \%$ de confianza.

\section{Resultados y discusión}

\subsection{Efectividad de los métodos de sincronización de estro}

En la figura 1 se observa el comportamiento de los resultados obtenidos en cuanto a la variable 

artificial transcervical en capra aegagrus hircus, Estelí 2020. Teknos Revista Científica, 21(2), 17-23.

efectividad de los métodos de sincronización de estro, de los datos tomados aplicando en la prueba de $T(p>$ $0.05)$ los resultados de los tratamientos no presentan diferencia estadística, el T2 con dispositivos CIDR reutilizados es superior al $\mathrm{T} 1$ con dispositivos CIDR nuevos, teniendo una diferencia del $100 \%$ de efectividad.

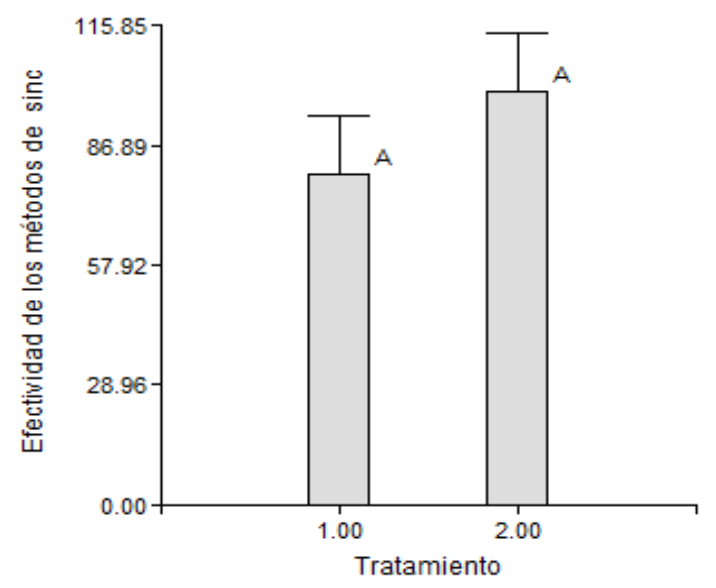

Figura 1. Efectividad de sincronización de celo

(Hernández, y otros, 2014), explican el "Efecto del reúso de dispositivos internos de liberación controlada de hormona en la sincronización y comportamiento reproductivo en cabras", donde se obtuvo como resultado un $100 \%$ en sincronización de estro. Para fertilidad el resultado fue $86,66 \%$ para $\mathrm{T} 1$ con dispositivos nuevos y $57,14 \%$ para $\mathrm{T} 2$ con dispositivos reutilizados. Para prolificidad se logró 1,62 y 1,88 crías en promedio para $\mathrm{T} 1$ y $\mathrm{T} 2$, respectivamente. Concluyendo que es posible inducir el estro en cabras con dispositivos reciclados sin afectar la prolificidad, sin embargo, la fertilidad disminuye cuando se utilizan por tercera vez.

Estos resultados son similares obtenidos en esta investigación.

Según (Velásquez, y otros, 2011) en su estudio "Reutilización del dispositivo de progesterona (CIDR) asociado con protocolos de corta duración en cabras", expresan en su investigación que es común que se reutilicen los CIDR para reducir costos, sin embargo, la fertilidad subsiguiente puede comprometerse; los resultados fueron una óptima sincronización del estro en la totalidad de los animales independiente del número de días del protocolo, los tratamientos cortos de seis días inducen efectivamente el estro durante la estación reproductiva resultando en alta fertilidad y se observó una disminución en la tasa de fertilidad en los protocolos usando el dispositivo reutilizado por 13 días.

Según los investigadores (Manes \& Ungerfeld, Sincronización de celos en ovejas y cabras con dispositivos intravaginales liberadores de progestágenos: alteraciones en ambiente vaginal y su relación con la fertilidad, 2015), en el estudio se manejaron teorías acerca de los dispositivos intravaginales en formas de esponjas impregnadas con progesterona o progestágenos. Los resultados de los protocolos para sincronización de celos fueron afectados no solo por la respuesta ovárica, sino también por los cambios generados en el ambiente vaginal, provocando una vaginitis en las cabras, estos cambios, son parcialmente responsables de la menor fertilidad obtenida con estos tratamientos con esponjas. Esta investigación se relaciona con el presente estudio ya que puede compararse con el T1.

\subsection{Porcentaje de hembras preñadas}

Se puede observar que en la figura 2, el porcentaje de hembras preñadas en promedio de cada uno de los tratamientos en el periodo evaluado, utilizando la prueba de $T(p<0.05)$, donde los resultados de los tratamientos no presentan diferencias estadísticas, siendo el T1 con dispositivos hormonales CIDR nuevos el que presentó mejores resultados con un $80 \%$, y el T2 utilizando dispositivos hormonales CIDR reutilizados con un $60 \%$ esto pudo deberse a la condición corporal de la cabra, así como el contenido hormonal.

Un estudio realizado por (López H., 2006) que trató de la "Evaluación de dos sincronizadores del celo en cabras primalas", donde se obtuvieron resultados en porcentaje de preñez de $97.67 \%$ y $8.33 \%$ de hembras vacías en el T1 con Lutalyse y un $83.33 \%$ de preñadas y un $16.67 \%$ de hembras vacias utilizando esponjas impregnadas con T2 progesterona, la fertilidad es 

artificial transcervical en capra aegagrus hircus, Estelí 2020. Teknos Revista Científica, 21(2), 17-23.

similar con el CIDR y con esponjas obteniendo un $63 \%$ en ambos casos, estos resultados son similares al del estudio presente.

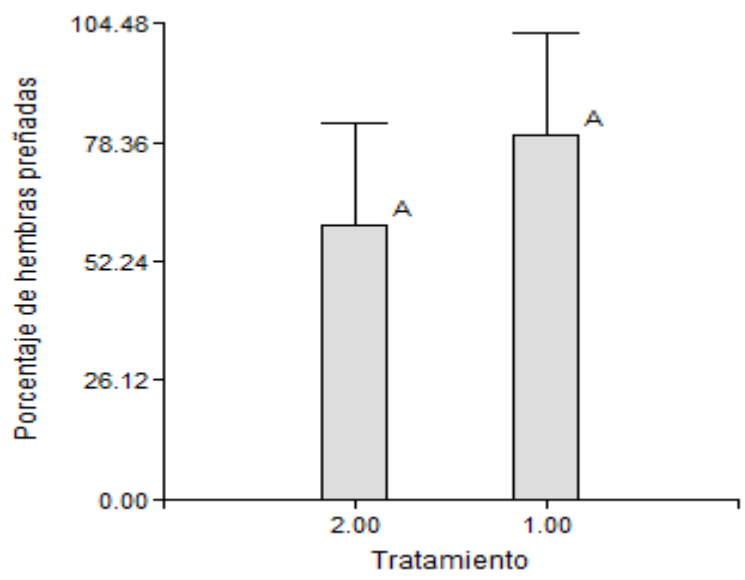

Figura 2. Porcentaje de hembras preñadas

Un estudio realizado por (López H., 2006) que trató de la "Evaluación de dos sincronizadores del celo en cabras primalas", donde se obtuvieron resultados en porcentaje de preñez de $97.67 \%$ y $8.33 \%$ de hembras vacías en el T1 con Lutalyse y un $83.33 \%$ de preñadas y un $16.67 \%$ de hembras vacías utilizando esponjas impregnadas con T2 progesterona, la fertilidad es similar con el CIDR y con esponjas obteniendo un $63 \%$ en ambos casos, estos resultados son similares al del estudio presente.

Un trabajo realizado en "Sincronización hormonal del estro en cabras criollas, bajo condiciones semiintensivas de Trópico seco en Nicaragua" por (Dávila \& G. Espinoza , 2012), donde se obtuvieron resultados de gestación en dos momentos: 45 y 60 días post retiro de dispositivos intrauterinos. El primer diagnóstico reveló que las hembras de los tratamientos $\mathrm{T} 1$ y $\mathrm{T} 2$, se encontraban en su totalidad gestantes; no así las del tratamiento T3, en donde el $70 \%$ estaban gestantes y un $30 \%$ se diagnosticaron como vacías. El segundo diagnóstico reveló que las hembras del T3 no incrementaron el porcentaje de preñez (70\%), en cambio, en los tratamientos T1 y T2 disminuyeron el número de hembras gestantes (en un 10 y 20\%, respectivamente), pudiendo estar relacionado a problemas de estrés por estabulación o problemas de observación al momento de realizar la revisión con ultrasonografía. Estos resultados no coinciden con los obtenidos en este estudio.

El estudio realizado por (Mogedas Moreno, 2016) "Sincronización de la ovulación y el ciclo inducido por el efecto "macho" mediante la administración de progesterona por vía intravaginal en cabras en período de anestro estacional", obteniendo los siguientes resultados: fertilidad en 228 cabras inseminadas, las cuales no mostraron diferencias significativas con ambos métodos utilizados, que fueron $57,25 \%$ intravaginal vs $48,8 \%$ intramuscular. En este estudio los resultados no son similares a la investigación realizada.

\subsection{Relación beneficio-costo}

En la variable relación beneficio-costo que se encuentra que en la tabla número 1, podemos comprobar el comportamiento de cada uno de los tratamientos en relación con cada uno de los indicadores financieros y productivos de la investigación, nos indica que el T1 resalta la confiabilidad en referencia al componente rentabilidad, este tratamiento refleja los mejores resultados con 1.04 , esto quiere decir que por cada dólar invertido se obtiene una ganancia de 0.04 centavos de dólar.

Tabla 1 Relación beneficio-costo

\begin{tabular}{llll}
\hline Tratamiento & Ingreso & Egreso & RCB \\
\hline 1 & 120 & 115 & 1.04 \\
2 & 90 & 95 & 0.95 \\
\hline
\end{tabular}

Según (Aguilera Díaz, 2017), el RCB nos indica que, se define como la relación entre los beneficios y los costos o egresos de un proyecto. Su cálculo se basa en la relación entre el valor actual de las entradas de efectivo futuras y el valor actual del desembolso original. Divide la corriente descontada de beneficios entre la de costos, por lo que este método también tiene en cuenta el valor del dinero en el tiempo. Explica que, si es mayor a uno, el aprisco obtiene ganancias, si es menor que uno tiene pérdidas y si se encuentra 
en uno no hay pérdidas ni ganancias, o sea, está en equilibrio.

Según (Castañer, 2014), hay diversos enfoques en el análisis de la relación beneficio-costo, pero todos en esencia tienen como objetivo el llevar al máximo posible la cuantificación de los beneficios y costos en términos monetarios. En el enfoque social, los costos y beneficios sociales podrán ser distintos de los contemplados por la evaluación privada económica.

En la variable beneficio-costo el $\mathrm{T} 1$ resalta la confiabilidad en referencia al componente rentabilidad, este tratamiento refleja los mejores resultados con 1.04 , esto quiere decir que por cada dólar invertido se obtiene una ganancia de 0.04 centavos de dólar, obtenido con el análisis RCB.

\subsection{Los resultados del análisis de la variable propuesta}

El plan de manejo reproductivo en UCATSE, muestra en conclusión, que se deben reestablecer ámbitos de manejo nutricional $y$ de algunas instalaciones para introducir la mejoría en la reproducción caprina utilizando los métodos de sincronización del estro e inseminación artificial, obteniendo así incremento eficaz y controlado en la producción del hato caprino.

\section{Conclusiones}

Al analizar las variables utilizadas en la investigación, se concluye que en lo referente a la variable efectividad de los métodos de sincronización no hubo diferencia estadística entre los tratamientos T1 con CIDR nuevo y T2 con CIDR reutilizado, los indicadores de efectividad fueron el aumento del tamaño del cérvix mediante la exploración con espéculo y fuente de luz, mucosidad blanquecina e incolora que indica aparición de la ovulación, así como también mediante la observación, se notaron cambios etológicos como el aumento de balar y en el movimiento de la cola que demuestran la efectividad de estro.
En cuanto a la variable porcentaje de preñez, no presentó diferencia estadística pero aritméticamente T1 presentó mejores resultados con un $80 \%$ de efectividad en comparación con el T2 que obtuvo un $60 \%$ ambos con inseminación artificial vía transcervical, en el T2 no se logró extraer suficiente semen del macho semental, por lo que dos hembras no fueron inseminadas artificialmente, los indicadores fueron el porcentaje de las crías en preñez mediante la palpación externa.

\section{Referencias}

Aguilera Díaz, A. (2017). El costo-beneficio como herramienta de decisión en la inversión en actividades científicas. Scielo. Obtenido de http://scielo.sld.cu/scielo.php?script=sci_arttext\&pid=S 2073-

60612017000200022\#: :text=Raz\%C3\%B3n\%20bene ficio\%2Fcosto\%20(B\%2F,valor\%20actual\%20del $\% 20$ desembolso\%20original.

Asociación Cooperativa La Galea. (2012). Sistema Integral de Manejo Caprino. Trujillo, Venezuela.

Castañer, J. (2014). Análisis del Costo Beneficio (Ejemplo de análisis en el sector privado). Obtenido de http://gis.jp.pr.gov/Externo_Econ/Talleres/Presentation CB_JP_ETI.pdf

Centro de seleccion y mejora génetica de ovino y caprino de Castilla y León. OVIGEN. (2010). Peculiaridades de la inseminación artificial caprina. Catilla y León: OVIGEN.

Contreras, I. (2008). Protocolo corto de sincronización de celo mediante la aplicación de cloprostenol y el uso del "efecto macho" en ovejas west african en condiciones tropicales. Tesis Doctorado, Universidad Completense de Madrid, Madrid.

Dávila, F., \& G. Espinoza , G. (2012). Sincronización hormonal del estro en cabras criollas, bajo condiciones semi-intensivas de Trópico Seco en Nicaragua. Managua: Universidad Nacional Agraria.

De la Rosa, S. (2011). Manual de Producción Caprina. Sangolqui: Formosa.

Enríquez Palos, J. L. (2017). Porcentaje de fertilidad de ovejas con estro sincronizado con CIDR reciclado y retirado en tres periodos de tiempo a través de inseminación artificial por laparoscopía. Toluca.

Galvan R., J. (1997). Efecto del CIDR-G y dos niveles de suplementación sobre la inducción del estro en cabras criollas en pastoreo . Marin, Nuevo León. 
Landero Amaya, J. A., Carrasco Ruiz, P. M., \& González, M. (2021). Evaluación de dos métodos de sincronización de estro e inseminación artificial transcervical en capra aegagrus hircus, Estelí 2020. Teknos Revista Científica, 21(2), 17-23.

García Agüero, K. M. (2018). Determinación de la tasa de presentación de celo y la tasa de concepción en cabras Saanen sincronizadas con acetato de medroxiprogesterona (MAP), durante dos épocas del año. Lima, Perú.

Gibbons, A., Cueto, M., \& Wolff, M. (2000). Manual de Inseminación Artificial en la Especie Caprina. Buenos Aires, Argentina.

Hernández, J., Navarrete, R., Alonso, M., Benítez, J., Gómez, A., Bernal, H., . . Orozco, M. (2014). Efecto del reuso de dispositivos internos de liberación controlada de hormona en la sincronización y comportamiento reproductivo en cabras. Nuevo León.

López H., S. (2006). Evaluación de dos sincronizadores del celo en cabras primalas . Coahuila.

Mafalda. (16 de Marzo de 2015). Capraispana. Obtenido de https://www.capraispana.com/la-inseminacion-encabras-proceso-de-realizacion/

Manes, J., \& Ungerfeld, R. (2015). Sincronización de celos en ovejas y cabras con dispositivos intravaginales liberadores de progestágenos: alteraciones en ambiente vaginal y su relación con la fertilidad. Revista Brasileira de Reprodução Animal - CBRA.

Manes, J., \& Ungerfeld, R. (2015). Sincronización de celos en ovejas y cabras con dispositivos intravaginales liberadores de progestágenos: alteraciones en ambiente vaginal y su relación con la fertilidad. Revista Brasil de Reproducción Animal.

Mogedas Moreno, M. (2016). Sincronización de la ovulación y el ciclo inducido por el efecto "macho" mediante la administración de progesterona por vía intravaginal en cabras en período de anestro estacional. Madrid.
Molina, P. (2005). Manipulación de la presencia del cuerpo lúteo en la sincronización del estro en ovejas Dorset. Agrociencia, 39: 11-18.

PerúLáctea. (02 de Junio de 2016). PerúLáctea. Obtenido de http://www.perulactea.com/2016/06/02/instalacionespara-una-buena-produccion-caprina/

Ruiz, R., Fernández, J. L., De La Vega, A. C., \& Rabasa, A. E. (2002). Evaluación de diferentes tratamientos hormonales para la sincronización del estro en cabras criollas serranas durante el verano. Tucumán: Portal Veterinaria.

Sáenz García, A. A. (2007). Ovinos y Caprinos. Managua: Universidad Nacional Agraria.

Salvatierra G., M., \& Contreras S., C. (2017). Manual de Producción Caprina. Instituto de Desarrollo Agropecuario- Instituto de Investigaciones Agropecuarias. Santiago, Chile: Instituto de Investigaciones Agropecuarias (INIA). Obtenido de https://www.inia.cl/wp-

content/uploads/ManualesdeProduccion/05\%20Manu al\%20Caprinos.pdf

Tenorio, V. (2010). Condiciones climáticas, Santa Adelaida, Estelí, Nicaragua. Esteli. Estelí: Instituto Nicaragüense de Estudios Territoriales, INETER.

Velásquez, L. F., Carolina, G., C. Ortiz, E., Izquierdo J., J., Lenz S., M., \& B., S. (2011). Reutilización del dispositivo de progesterona (CIDR) asociado. vetzootec, 39-46.

Zoetis. (2018). CIDR Ovis. Obtenido de https://www.zoetis.es/_locale-assets/spc/cidr-138-gdispositivo-vaginal-para-vacas.pdf 